\title{
Unilineage Model of Hematopoiesis Predicts Self-Renewal of Stem and Progenitor Cells Based on Ex Vivo Growth Data
}

\author{
Ching-An Peng, ${ }^{1}$ Manfred R. Koller, ${ }^{1,2}$ and Bernhard Ø. Palsson ${ }^{1 *}$ \\ 'Department of Chemical Engineering, University of Michigan, Ann Arbor, \\ Michigan; and ${ }^{2}$ Aastrom Biosciences, Inc., Ann Arbor, Michigan
}

Received June 1, 1995/Accepted March 21, 1996

\begin{abstract}
Stem cell models are used to describe the function of several tissues. We present unilineage kinetic description of stem cell models and their application to the analysis of ex vivo hematopoietic cell expansion data. This model has the capability to simulate the total cell number and the number of cells at each stage of differentiation over time as a function of the stem cell self-renewal probability, the growth rate of each subpopulation, and the mature cell death rate. The model predicts experimental observations in perfusion-based hematopoietic bioreactor systems. To obtain net cell expansion ex vivo, the model simulations show that the stem cell self-renewal probability must exceed one-half, thus resulting in net expansion of the stem cell population. Experimental data on longterm culture-initiating cells (LTC-IC) confirm this prediction and the probability of self-renewal is estimated to be 0.62 to 0.73 . This self-renewal probability, along with the death rate, define a relationship in which the apparent overall growth rate is less than the compartmental growth rate. Finally, the model predicts that cells beyond the stem cell stage of differentiation must self-renew to achieve the level of expansion within the time frame observed in experimental systems. (c) 1996 John Wiley \& Sons, Inc. Key words: unilineage model $\cdot$ tissue function ex vivo • hematopoiesis • stem cell expansion
\end{abstract}

\section{INTRODUCTION}

Actively proliferating tissues in vivo are believed to generate mature cells through a complex differentiation process that originates from an undifferentiated cell called the stem cell. This process is perhaps best documented for bone marrow (Emerson, 1991; Hall and Watt, 1989; Heimfeld and Weissman, 1991), epidermis (Hall and Watt, Potten et al., 1982; Wright, 1983), villi formation in the small intestine (Hall and Watt, 1989; Potten and Loeffler, 1987, 1990), and has more recently been suggested for liver (Sell, 1990; Sigal et al., 1992; Thorgeirsson, 1993) and neural crest (Davis and Temple, 1994; Stemple and Anderson, 1992). Kinetic models of cell growth, sometimes accounting for underlying me-

\footnotetext{
* To whom all correspondence should be addressed at: Department of Bioengineering, 9500 Gilman Drive, Mail Code 0412, University of California at San Diego, La Jolla, CA 92093-0412. Telephone: 619534-5668; fax: 619-822-0240; e-mail: palsson@ucsd.edu
}

tabolism and other biochemical processes (Nielsen and Villadsen, 1992) have played an important role in the study and use of continuous, homogeneous cell lines and strains. As the field of tissue engineering develops, kinetic models are likely to play an important role in the development of reconstituted ex vivo tissue functions and in the study of the fate of cells in vivo following transplantation. However, because tissues are inherently more complex than homogeneous cell cultures, the kinetic models will be considerably more complex, needing to incorporate stem cell self-renewal, differentiation, and cell to cell interactions. Furthermore, tissues in which the geometric organization is important for cell growth and function (e.g., the small intestine) will require an addition level of complexity.

Compared with other tissues, bone marrow has been relatively well studied, and the hematopoietic process has been the subject of model-building in a number of studies (Nakahata et al., 1982; Schmitz et al., 1993; Schofield et al., 1980; Till et al., 1964; Vogel et al., 1968; Warner and Athens, 1964; Wichmann et al., 1988). Also, bone marrow cultures have significant applications to clinical practice and basic scientific studies of tissue structure-function relationships (Koller and Palsson, 1993). Therefore, hematopoiesis is a logical candidate system for the initial development of mathematical models of ex vivo tissue function. The first step toward this goal is presented in this study.

Long-term bone marrow cultures (LTBMC) were developed in the 1970s for the murine system (Dexter et al., 1977). Unfortunately, human LTBMC have not performed as well as their murine counterparts, despite extensive research in this area (Eaves et al., 1991; Greenberg, 1984). Prolific human hematopoietic culture conditions have been developed that are based on rapid medium replacement to mimic the dynamic in vivo environment (Schwartz et al., 1991a, 1991b). Based on these culture conditions, a continuous perfusion bioreactor system has been developed that supports much higher cell densities than those obtained under static tissue culture protocols (Koller et al., 1993a, 1993b). These and related developments have been reviewed in 
detail (Koller and Palsson, 1993). While expansion of cells at all stages of differentiation is obtained in these bioreactors, it is likely that the performance of these bioreactor systems can be further optimized. A dynamic model of stem cell self-renewal and differentiation is required to conceptualize events taking place in the bioreactors, to predict and seek consistency in the observed data, and eventually to be used for optimization of bioreactor performance. In the present study, we develop a unilineage kinetic model of blood cell formation that is based on the biological stem cell model for hematopoiesis.

\section{DESCRIBING TISSUE FUNCTION WITH UNILINEAGE KINETIC MODELS}

The function of many tissues is conceptually described by a stem cell model (Caplan, 1991; Emerson, 1991; Hall and Watt, 1989; Warner and Athens, 1964; Wichmann et al., 1988; Wright, 1983). According to this model, all mature parenchymal cells originate from a pluripotent stem cell. Stem cells are endowed with the capability to self-renew and thus maintain their phenotype indefinitely. A daughter cell resulting from a stem cell division may undergo commitment and differentiation, thereby losing its stem cell properties. The committed cell then undergoes a proliferation and differentiation process to generate a large number of mature parenchymal cells. In bone marrow this process is called hematopoiesis, a process that will be considered in what follows.

\section{Model Assumptions and Dynamic Description}

If one is only concerned with total cell production in an ex vivo bone marrow system, and not the number of cells at each stage of differentiation in each lineage, one can describe the hematopoietic process with a unilineage model (Warner and Athens, 1964). The pathways for cell growth and self-renewal of stem cells according to this scheme are depicted in Figure 1. A kinetic description of this model is based on the following assumptions:

1. The hematopoietic process is viewed as a series of discrete stages through which the cells pass as they differentiate.

2. The stem cells are in the first compartment. Upon division, a stem cell may self-renew or differentiate (Till et al., 1964). The probability of self-renewal, $f$, is between 0 and 1 . If differentiation occurs, the cell

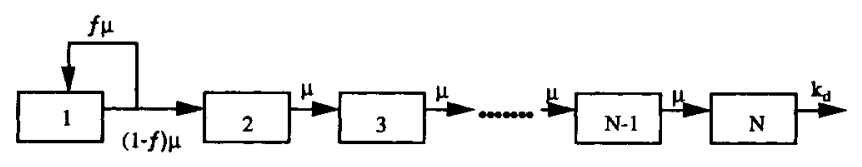

Figure 1. A schematic representation of a unilineage cell kinetic model of hematopoiesis. moves on to the next stage of differentiation, otherwise, it remains in the stem cell compartment.

3. The cell specific growth rate, $\mu$, accounts for the maximum cell density $\left(x_{\max }\right)$ that is observed in bioreactors (Koller et al., 1993b; Palsson et al., 1993):

$$
\mu=\mu_{\max }\left(1-\frac{\sum_{i=1}^{N} x_{i}}{x_{\max }}\right)
$$

where the cell density is denoted by $x$, the differentiation stage by $i$, and the maximal growth rate is $\mu_{\max }$. When $x \ll x_{\max }$, the increase in cell number is exponential; when $x$ approaches $x_{\max }$ the growth rate is attenuated, and the cell density approaches its maximum value.

4. Two further simplifying assumptions are made: the effects of mitogens are constant, thus not influencing the growth rates; and also, growth rates are the same for cells of all stages of differentiation. Relaxing these assumptions would lead to more complex models that contain parameters that cannot be readily estimated with presently available experimental data.

5. The last compartment containing the fully mature cells has a death rate of $k_{d}$ representing the continuous turnover of most mature blood cells. Cell death in intermediate compartments may occur, especially if an insufficient amount of growth factors is present.

Based on these simplifying assumptions, a cell population balance on each compartment in Figure 1 leads to a set of dynamic equations that describe the hematopoietic process:

$$
\begin{aligned}
\frac{d x_{1}}{d t} & =(2 f-1) \mu x_{1} \\
\frac{d x_{2}}{d t} & =2(1-f) \mu x_{1}-\mu x_{2} \\
\frac{d x_{n}}{d t} & =2 \mu x_{n-1}-\mu x_{n} \quad n=3,4, \ldots, N-1 \\
\frac{d x_{N}}{d t} & =2 \mu x_{N-1}-k_{d} x_{N}
\end{aligned}
$$

with initial conditions:

$$
\left.x_{i}=x_{i}\right)_{o}, \quad i=1,2, \ldots, N, \quad @ t=0
$$

\section{Scaling and Dimensionless Kinetic Equations}

Logical reference scales for time and cell density are the maximum growth rate and the maximum cell density, respectively. Eqs. (2)-(6) are made dimensionless by introducing the following variables:

$$
\tau=\mu_{\max } t x_{i}^{*}=\frac{x_{i}}{x_{\max }} ; x_{s u m}^{*}=\sum_{i=1}^{N} x_{i}^{*}
$$


$x_{\max }$ is the maximum cell density. The dimensionless dynamic equations therefore become:

$$
\begin{aligned}
\frac{d x_{1}^{*}}{d \tau} & =(2 f-1)\left(1-x_{\text {sum }}^{*}\right) x_{1}^{*} \\
\frac{d x_{2}^{*}}{d \tau} & =2(1-f)\left(1-x_{\text {sum }}^{*}\right) x_{1}^{*}-\left(1-x_{\text {sum }}^{*}\right) x_{2}^{*} \\
\frac{d x_{n}^{*}}{d \tau} & =\left(2 x_{n-1}^{*}-x_{n}^{*}\right)\left(1-x_{\text {sum }}^{*}\right) \quad n=3,4, \ldots, N-1 \\
\frac{d x_{N}^{*}}{d \tau} & =2\left(1-x_{\text {sum }}^{*}\right) x_{N-1}^{*}-\frac{k_{d}}{\mu_{\max }} x_{N}^{*} \\
x_{i}^{*} & \left.=x_{i}^{*}\right)_{o}, \quad i=1,2,3, \ldots, N, \quad @ \tau=0
\end{aligned}
$$

This system of ordinary differential equations can be integrated to determine the cell distribution at the various stages of differentiation as functions of time and the kinetic parameters of the model.

\section{Model Parameters and Estimated Numerical Values}

The parameters appearing in the model are $f, \mu_{\max }, k_{d}$, $x_{\max }$, and $N$. To carry out the simulations, the initial cell density in each compartment must be specified. The apparent regeneration time for hematopoietic cell populations in vivo is about 2 days (Koller and Palsson, 1993), and this time is closely approximated in the perfusionbased bioreactor systems. Thus, a representative value for the maximal growth rate is $\mu_{\max }=0.35$ day $^{-1}$. Faster growth rates have been reported for purified primitive bone marrow cells (characterized by the presence of the CD34 surface antigen [Civin et al., 1984] and the lack of CD38, HLA-DR, or CD71). The reported doubling times for this population can be calculated from published cell expansion data and they fall in the range of 0.76 to 1.0 days (Lansdorp and Dragowska, 1992; Mayani et al., 1993) to 1.6 to 2.1 days (Brandt et al., 1992; Srour et al., 1993).

The death rate in the last compartment depends upon the mature cell type. For instance, mature erythrocytes have a lifespan of 120 days, whereas granulocytic cells have a lifespan of less than 1 day (Cronkite, 1988). Therefore, in the simulations presented below, we show the effect of several different death rates.

Perhaps the most important parameter of this model is the self-renewal probability, $f$. Under steady-state in vivo conditions, $f$ must be 0.5 . However, for ex vivo expansion of hematopoietic cells, $f$ should exceed onehalf, resulting in an expansion of the stem cell population (shown in Fig. 2).

The number of differentiation steps can be estimated from experimental data on long-term culture-initiating cell (Eaves et al., 1992; Moore, 1991; Ploemacher et al., 1991). According to reported densities of LTC-IC in bone marrow, primitive cell frequencies are on the order of 1 per 20,000 (Sutherland et al., 1990). Assuming that bone marrow is in a balanced differentiation stateexponentially increasing with compartment number in accord with the growth and death rate-we can estimate that the total number of proliferative stages, $N$, is approximately 14 (i.e., $2^{14}$ is approximately 20,000 ). To compare our model with ex vivo growth data, we will assume for the present purposes that the LTC-IC assay detects stem cells and that the LTC-IC density can be used to estimate the length of the differentiation pathway. However, it is important to note that while the LTC-IC assay measures very primitive cells (Eaves et al., 1992), the LTC-IC population is somewhat heterogeneous (Sutherland et al., 1990) containing cells with different degrees of proliferative potential. Furthermore, other methods to estimate the number $N$ have been presented (Schmitz et al., 1993).

The maximum cell density, $x_{\max }$, is a property of the culture system used. The reported value for $x_{\max }$ in a hematopoietic perfusion bioreactor system has been reported to be roughly 3 million cells $/ \mathrm{cm}^{2}$ (Koller et al., 1993b; Palsson et al., 1993). This value will vary with the culture system used.

Initial conditions must be assigned. Two types of initial conditions are of interest. First, an exponential distribution of cells with more cells in later compartments simulating balanced differentiation in vivo, and second, a single cell in one compartment simulating the kinetics of colony-forming assays.

\section{Solution Method}

The model system just formulated is readily solved using any standard numerical method that integrates a set of ordinary differential equations. For the computations presented here, the Runge-Kutta fourth-order method (Carnahan et al., 1969) was used to integrate the differential equations.

\section{RESULTS AND DISCUSSION}

The kinetic model can be used quantitatively to examine the interactions of the parameters which it embodies, such as the effects of self-renewal and death rate on cell production. It can also be used to estimate kinetic parameters from experimental data. The model can predict total cell production and the number of cells at all stages of differentiation, including stem cells. We now use the model to address a series of questions.

\section{What Is the Effect of Self-Renewal Probability and Death Rate on Cell Production?}

We first examine the influence of the kinetic parameters, 

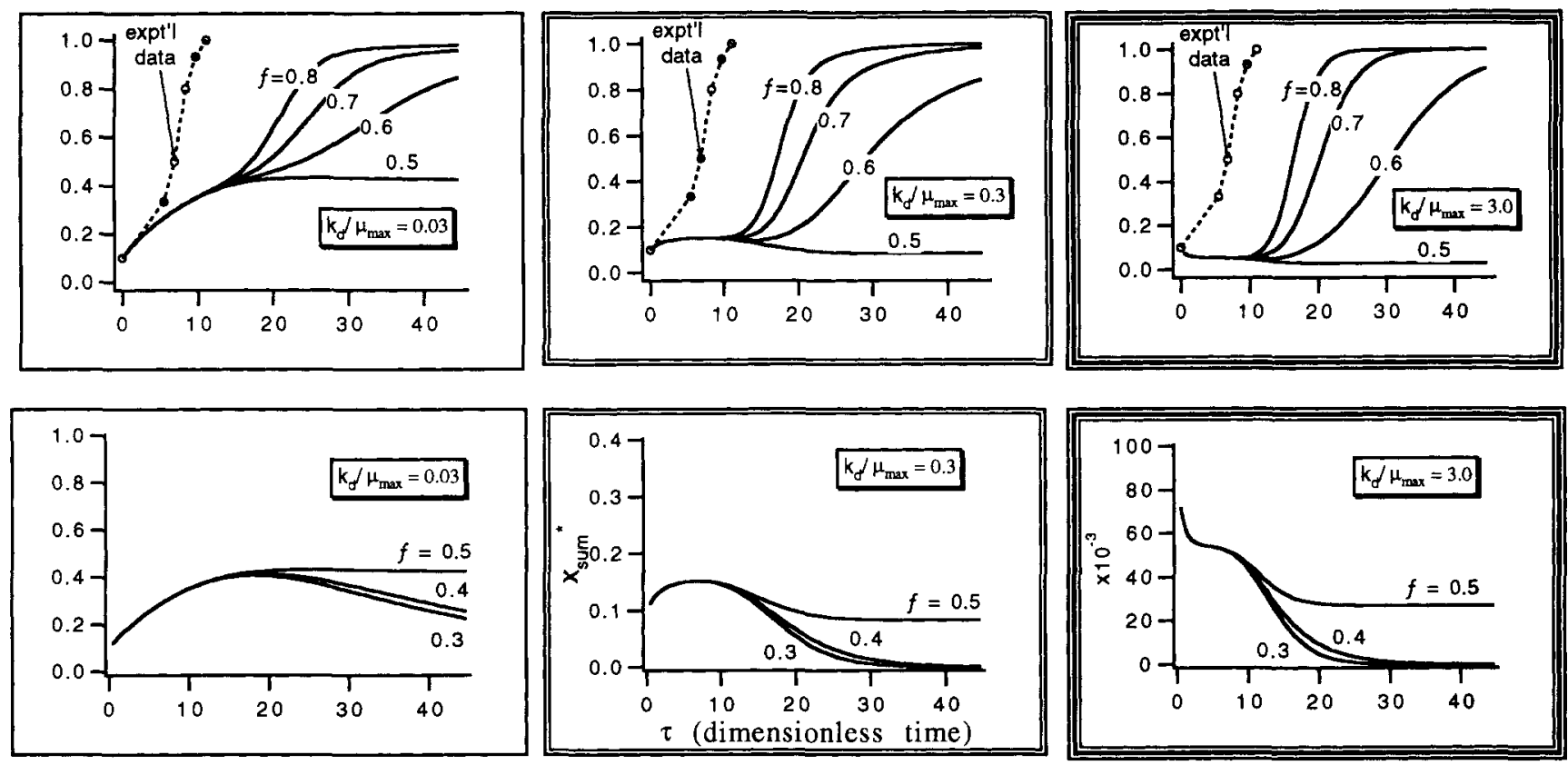

Figure 2. Simulated growth characteristics for different stem cell self-renewal probabilities and mature cell death rates. The three columns are for various ratios of death rate to growth rate. The upper row of panels is for self-renewal, $f$, exceeding one-half, and the lower row for $f$ less than one-half. Note the dotted line is the experimental data adopted from Figure 1 of Koller et al. (1993b) for comparison using $\mu_{\max }$ that corresponds to a doubling time of 1 day.

nificant effect on cell production is exerted by $f$ (Fig. 2). In spite of the influence of $k_{d} / \mu_{\max }$ on the growth curves, it is clear that $f$ must be greater than one-half to obtain net expansion of total cell numbers over time. Thus, we conclude that, if the stem cell model holds, the cell expansion observed in perfused cultures (Koller et al., 1993b; Palsson et al., 1993) indicates a self-renewal probability exceeding 0.5 under these conditions.

Three different dimensionless death rates for the last mature compartment $-0.03,0.3$, and 3.0-were used in the simulation. If $\mu_{\max }$ is 0.35 per day, these values of $k_{d} / \mu_{\max }$ correspond to the half-life of cells in the last compartment being 1600,160 , and $16 \mathrm{~h}$, respectively. It should be noted that for granulocytes the ratio $k_{d} / \mu_{\max }$ is greater than unity, whereas for erythrocytes it is much smaller than unity.

\section{What Is the Predicted Self-Renewal Probability Based on Experimental Data?}

Next we obtain a quantitative estimation of the value of $f$ based on reported experimental data. The effect of $f$ on the cell number in the first compartment is shown in Figure 3. As expected, when $f=0.5$, the cell number in the first compartment is time invariant. After division, one of the daughter cells remains in the stem cell compartment, while the other differentiates into the next compartment. Above 0.5, the cell number in the stem cell compartment increases as the value of $f$ is increased. Experimental data for the stem cell compartment, as determined by the LTC-IC assay, indicate an average 7.5-fold expansion during a 14-day cultivation period (Koller et al., 1993b). The numerical value for $f$ can be estimated by using dimensionalized time $\tau\left(=\mu_{\mathrm{max}} t\right)$. For a 14-day culture, we can estimate the value of $\mu_{\max } t$ for possible doubling times:

$$
\begin{aligned}
& \mu_{\max }=10 \quad \text { if the doubling time is } 1 \text { day; and } \\
& \mu_{\max } t=5 \quad \text { if the doubling time is } 2 \text { days }
\end{aligned}
$$

Thus, $\mu_{\max } t$ must fall in the range of 5 to 10 for likely doubling times in the stem cell compartment. Drawing a line on Figure 3 for an expansion ratio of 7.5 , one estimates that $f$ is in the range of 0.62 to 0.73 if $\mu_{\max } t$ is in the range of 5 to 10 .

About three decades ago, a stochastic model of stem cell proliferation based on the growth of murine spleen colony-forming units (CFU-S) was developed (Till et al., 1964). The self-renewal probability of the murine CFU-S was estimated to be 0.6 using Monte Carlo simulation of experimental data. After this model was proposed, several investigators estimated the self-renewal capability of the CFU-S. For example, the self-renewal of CFU-S was measured through serial transplantation (Schofield et al., 1980). Based on a previously developed statistical method (Vogel et al., 1968), it was concluded that, for normal murine bone marrow, the mean selfrenewal probability was 0.683 . Later, the theory of branching processes was employed to investigate the self-renewal of stem cells and production of CFU. GEMM by replating individual colonies in culture (Nakahata et al., 1982). The self-renewal probability for 

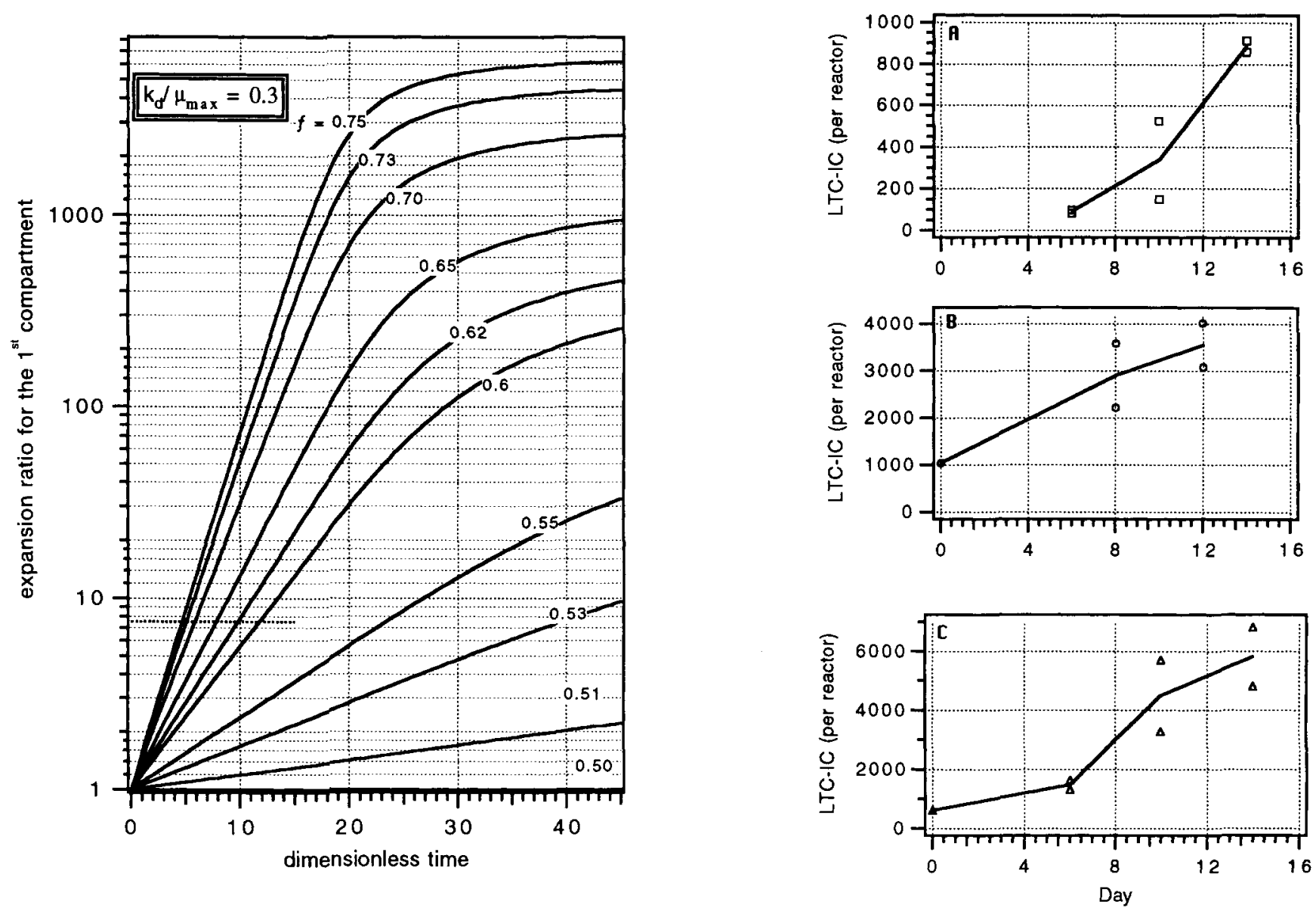

Figure 3. (a) Simulated expansion in the first compartment for varying values of the self-renewal probability, and (b) experimental data for LTC-IC expansion (data replotted from Koller et al., 1993b). Here the parallel datapoints are the duplicated measurements.

CFU-S was estimated to be 0.589 , which is consistent with the estimate from the stochastic model of CFU-S renewal (Till et al., 1964). Cell population kinetics, such as those used herein, have been used to investigate the mechanism of stem cell self-renewal and commitment based on CFU-S data (Wichmann et al., 1988). This model accounted for the stem cell pool and a few simplified compartments of differentiated cells. The self-renewal probability was estimated to be approximately 0.6. However, it should be noted that the murine CFU-S assay is no longer thought to measure pluripotent hematopoietic stem cells (Koller and Palsson, 1993).

\section{What Is the Relationship Between the Population and Individual Growth Rates?}

Based on the LTC-IC expansion data, we obtain a value for $f$ that is approximately 0.62 to 0.73 . Based on the overall growth data, the population doubling time is about 2 to 2.5 days (Koller et al., 1993b; Palsson et al., 1993). These two observations seem inconsistent with the overall growth observed in the perfusion cultures over 14 days and the simulations presented in Figure 2; namely, not enough total cells are generated within about 5 to 10 dimensionless time units. Thus, the question arises: What is the relationship between the overall population growth rates and the growth rates of the individual cell compartments? To help answer this question, we obtain the dynamic equation for the whole population by summing up Eqs. (2)-(5):

$\frac{d x_{\text {sum }}}{d t}=\mu\left[1-\left(1+\frac{k_{d}}{\mu}\right) \frac{x_{N}}{x_{\text {sum }}}\right] x_{\text {sum }}=\mu_{\text {app }} x_{\text {sum }}$

The apparent growth rate $\left(\mu_{\text {app }}\right)$ is thus related to the compartmental growth rate $(\mu)$ by:

$$
\mu_{a p p}=\mu\left[1-\left(1+\frac{k_{d}}{\mu}\right) \frac{x_{N}}{x_{s u m}}\right]
$$

Thus, the apparent growth rate will be lower than the compartmental growth rate. The difference between the apparent and the compartmental growth rate depends on the death rate and the fractional cell number, $x_{N}$ ' $x_{\text {sum }}$, in the last compartment.

To illustrate this effect by simulation, we compute the outgrowth from a single stem cell and compute $x_{N} /$ $x_{\text {sum }}$ as a function of time for various self-renewal probabilities (Fig. 4). The relationship between $f$ and $x_{N} / x_{\text {sum }}$ 


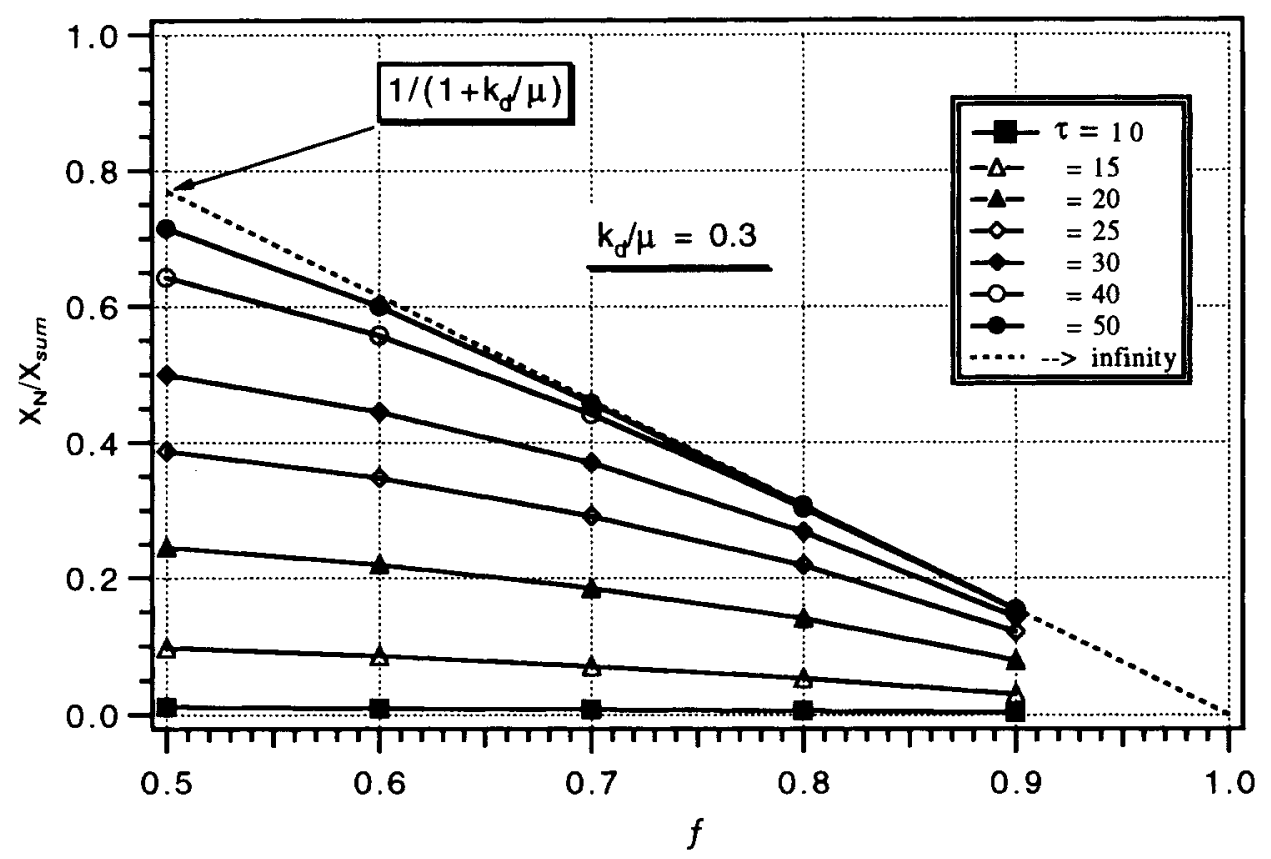

Figure 4. The effect of self-renewal probability, $f$, on the fraction of total of cells in the last compartment of differentiation. At long time intervals this ratio has a linear relationship with $f$.

is linear after a sufficiently long time for $f$ values of 0.5 to 1.0. One can derive an analytical form for this line by solving for the steady-state solution of Eqs. (2)-(5) for $f=0.5$ :

$$
\frac{x_{N}}{x_{\text {sum }}}=\frac{2(1-f)}{\left(1+k_{d} / \mu\right)}
$$

At long time intervals, after all stages of differentiation have been populated, the relationship between the apparent and compartmental growth rate becomes:

$$
\frac{\mu_{a p p}}{\mu}=2 f-1
$$

The difference between the apparent and compartmental growth rates thus depends on the self-renewal probability. If $f=0.5$, the overall growth rate is 0 , and if $f<$ 0.5 , the eventual growth rate is negative as the cell population disappears (Fig. 5). If the self-renewal probability is 0.62 to 0.73 , the apparent growth rate is about $24 \%$ to $46 \%$ of the compartmental growth rate. Thus, the 2- to 2.5-day population doubling time could be a factor of $2.2(f=0.73)$ to $4.2(f=0.62)$ longer than the compartmental doubling time. Even if the compartmental doubling time is as low as 0.65 day and $\tau\left(=\mu_{\max } t\right)$ is 15 , the overall computed cell growth according to the stem cell model is not consistent with experimental data (Koller et al., 1993b) (see Fig. 2, top row). The insufficient cell production over the possible time period leads us to the next question.

\section{Do Progenitor Cells Self-Renew?}

Even though the apparent and compartmental growth rates differ significantly, the maximum cell density may not be reached within 14 days with the model thus far presented. Cell production would be increased if there was self-renewal at other stages of differentiation in addition to the stem cell compartment. Furthermore, as discussed below, literature data indicate that selfrenewal of progenitor cells is required to obtain the experimentally observed cell growth.

Replication and differentiation are often thought of as two orthogonal processes. Renewal of stem or progenitor cells can be pictured schematically on a twodimensional diagram where the $x$-axis denotes the differentiation stage (or the compartment number) and the $y$-axis the number of replications that take place

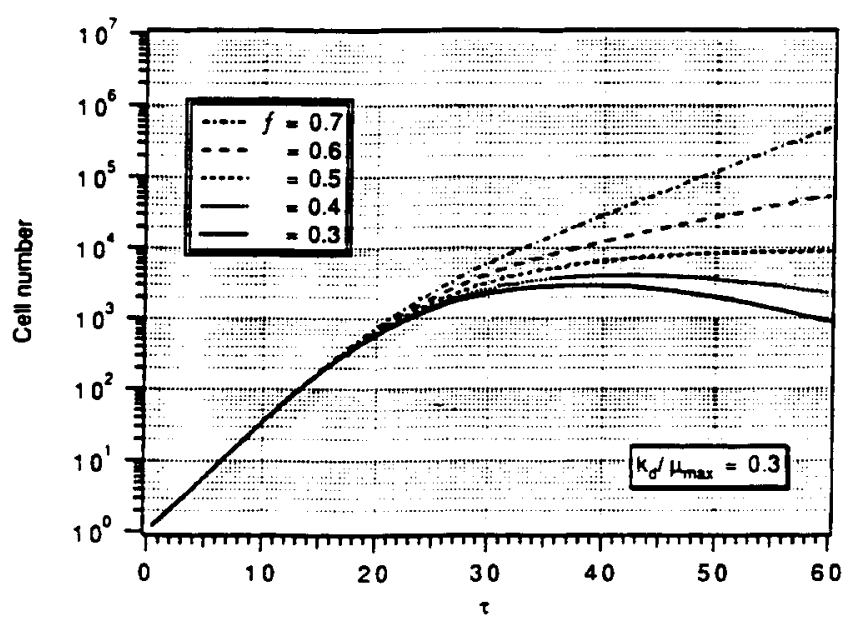

Figure 5. Simulation of the outgrowth from a single LTC-IC for varying self-renewal probabilities. The intrinsic and apparent growth rates differ once all stages of differentiation are populated. The growth rate following balanced differentiation depends only on $f$. 


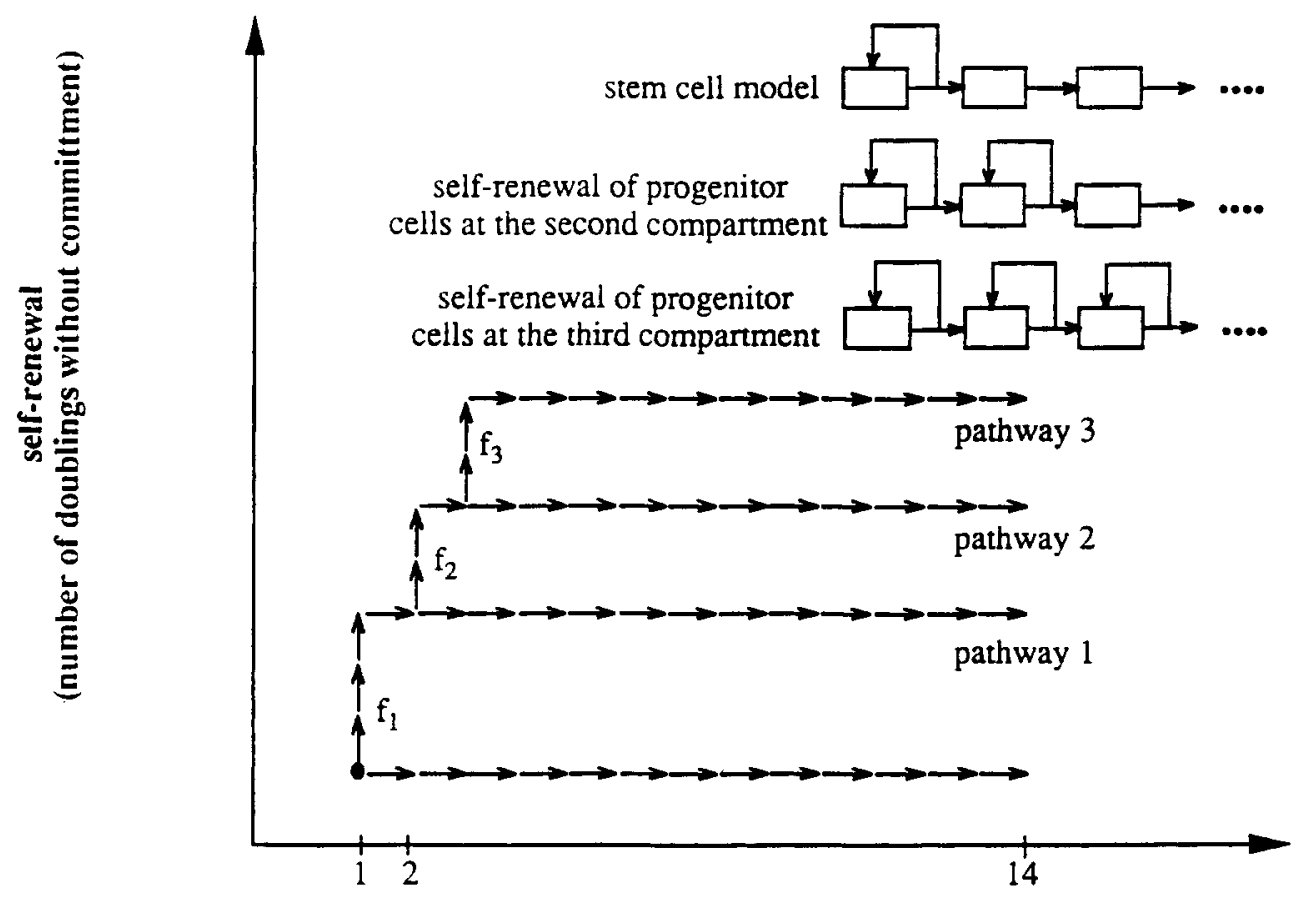

number of differentiating compartment

Figure 6. Schematic representation of the paths of stem cell self-renewal and differentiation. The two axes represent cell doublings without differentiation ( $y$-axis) and with differentiation ( $x$-axis). The three pathways represent possible self-renewal and differentiation paths based on the compartmental models shown. Pathway 3 will generate many more mature cells than pathway 1, even though the number of differentiation steps is the same.

within a compartment before further differentiation (Fig. 6). For illustration, the figure shows the paths that cell growth would follow for self-renewal in either the first compartment (the stem cell model), in both the first and second compartments, or in the first three compartments.

Simulation of total cell production where self-renewal takes place in other compartments in addition to the first one shows that the overall cell production can be significantly increased (Fig. 7). By assigning $f$ of the stem cell compartment to be 0.7, Figure 7a shows the effect of self-renewal in the second compartment on the total cell number. It shows that the time required to approach maximum cell density can be shortened by increasing self-renewal in the second compartment. The possible shift in time is limited and requires self-renewal probability in the second compartment that exceeds 0.5 .

Increasing the number of compartments that display self renewal will shift the growth curve even further to the left. With self-renewal for the first compartment of 0.7 , and for an increasing number of subsequent compartments with self-renewal of 0.5 , Figure $7 \mathrm{~b}$ shows that the cell production can be greatly enhanced over short time intervals. The experimental growth data from the bioreactors can thus be simulated if non-stem cell compartments display a moderate degree of self-renewal.
The stem cell model can represent all the observed cell production data obtained from the perfusion bioreactors if self-renewal is not confined to the stem cell compartment. Is this result consistent with the experimental literature? Several lines of investigation indicate that the answer is yes:

1. As detailed previously, in vivo data from the murine system provides evidence for self-renewal in the CFU-S compartment. Because CFU-S are no longer considered to be stem cells, this result now suggests that a nonzero self-renewal probability can be assigned to a progenitor compartment.

2. In vitro replating experiments of human progenitor cells has demonstrated some degree of self-renewal capacity. Umbilical cord blood multipotential progenitors (CFU-GEMM) were found to have extensive self-renewal capability (Carow et al., 1993). About half of these primary colonies, which contained roughly $10^{5}$ cells, were capable of generating an average of 80 secondary colonies containing a total of about $10^{6}$ cells. Replating of human high proliferative potential colony-forming cell (HPPCFC) colonies has similarly shown the ability of about one-half of the colonies to generate multiple secondary colonies (Srour et al., 1993). 


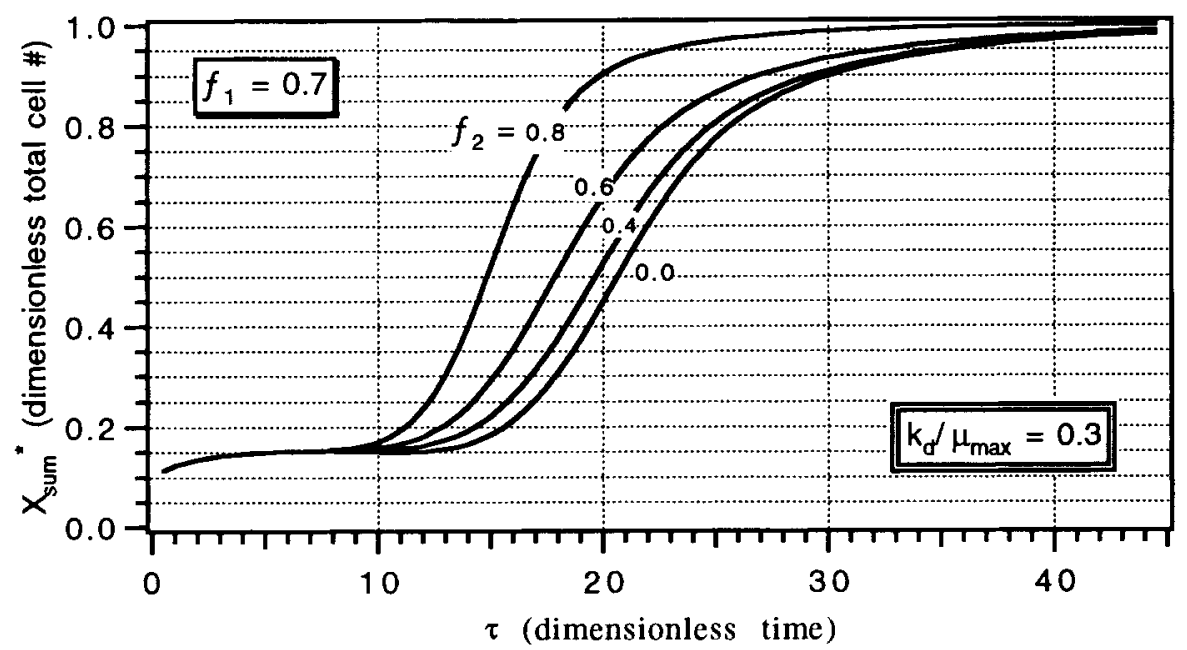

(a)

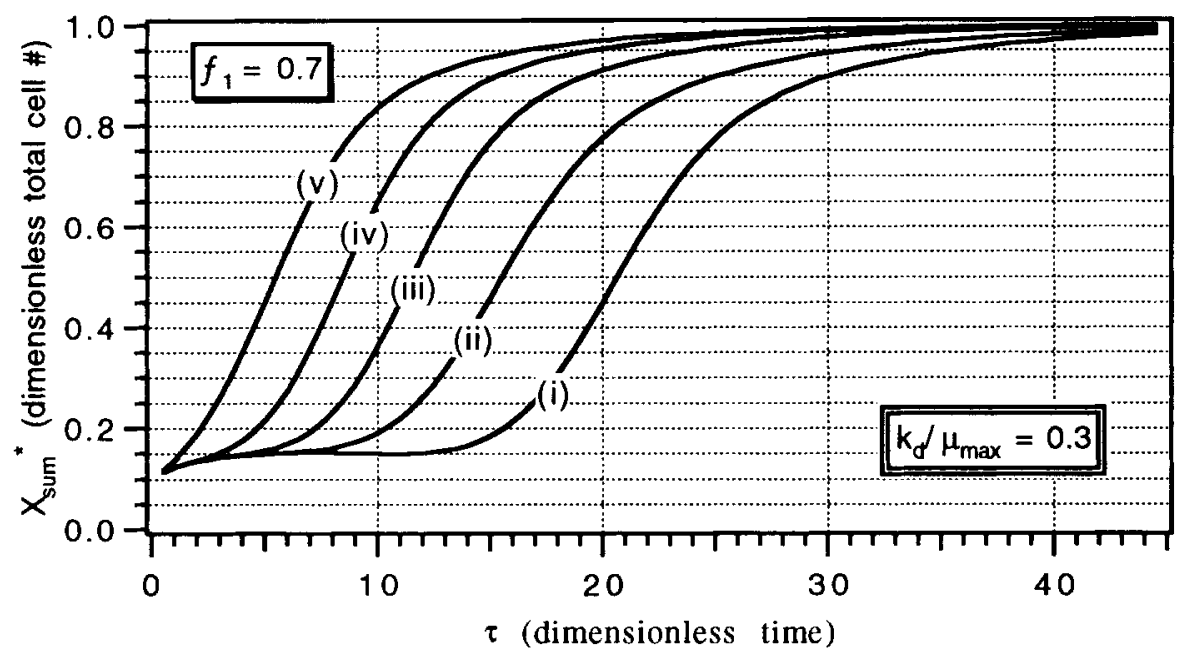

(b)

Figure 7. (a) The effect of self-renewal in the second compartment on cell expansion, and (b) the effect of self-renewal of 0.5 in an increasing number of non-stem cell compartments: (i) self-renewal in stem cell compartment only; (ii) compartments 2 to 4 self-renew; (iii) compartments 2 to 7 self-renew; (iv) compartments 2 to 10 self-renew; and (v) compartments 2 to 13 self-renew. In all cases, the self-renewal probability of the first compartment is 0.7 .

3. The aforementioned reports on the large size of certain clonal colonies, especially after replating (see above), suggest many cell doublings. The 20 doublings required to generate $10^{6}$ cells from a single non-stem cell (CFU-GEMM) clone (Carow et al., 1993 ) is not consistent with the number of differentiation steps that exist between the CFU-GEMM and the mature cell stage. Therefore, this result implies that some progenitor cells must undergo selfrenewal.

Taken together, the data from the experimental literature and the simulations presented herein argue for the occurrence of self-renewal in non-stem cell compartments. This result is an important departure from the generally accepted stem cell model.

The model presented in this study is formulated using several restrictive simplifying assumptions. Models of lineage development need to be further developed. Extension to account for the effects of specific growth factors would be important. This extension would require functional information about the effects of growth factors on the different compartments and the source of such growth factors. Growth factors can either inhibit or stimulate differentiation and replication. Once these 
relationships have been elucidated, a lineage model can be used to examine the fate of specific subpopulations in the hematopoietic hierarchy. Maximizing the production of the myeloid progenitor subpopulation would be of particular interest, because these cells are believed to play a key role in the engraftment process that follows transplantation.

Finally, it has been recently shown that significant concentration gradients of secreted growth factors and chemotactic factors can exist in commonly used bioreactor configurations (Peng and Palsson, 1995, 1996). These concentration gradients can lead to spatial dependency of growth. Therefore, it is likely that accurate simulation of replication and differentiation in bioreactors will require the simultaneous consideration of biological and physicochemical rate processes. Models that describe the dynamics of lineage development, such as that presented in the present study, will constitute boundary conditions in integrated models of tissue function in tissue-engineered systems.

\section{CONCLUSIONS}

The unilineage kinetic model, based on the currently accepted stem cell model of hematopoiesis, predicts that a stem cell self-renewal probability of greater than 0.5 is required for obtaining net cell expansion. This finding is of great importance for clinical use of human bone marrow cell populations expanded ex vivo, and LTCIC data are consistent with this prediction (Koller et al., 1993b). The estimated range of the self-renewal probability of LTC-ICs is between 0.62 and 0.73 , which is consistent with estimates based on mathematical analyses and experimental data of the murine hematopoietic system. The number of stages estimated based on LTCIC data are inconsistent with the number of cells that can be obtained from progenitor cells in vitro, as is the total cell production calculated from the stem cell model and experimental data from human bone marrow perfusion cultures. This points to a shortcoming of the most commonly accepted stem cell model of hematopoiesis. If self-renewal is confined to the stem cell compartment, then the number of stages in the differentiation process is much greater than that estimated based on LTC-IC data. Alternatively, there exists a significant degree of self-renewal at progenitor or pre-progenitor stages of differentiation. Growing experimental data points to the latter possibility.

This work was supported by a grant from Aastrom Biosciences, Inc., Ann Arbor, MI.

\section{NOMENCLATURE}

$\begin{array}{ll}f & \text { probability of self-renewal } \\ \mathrm{i} & \text { differentiation stage } \\ k_{d} & \text { death rate of the last compartment }\end{array}$
$N \quad$ number of total compartments

$n \quad$ specific compartment

$t$ time

$t_{d} \quad$ doubling time

$\tau$ dimensionless time

$\mu \quad$ compartmental growth rate

$\mu_{\text {app }}$ apparent overall growth rate

$\mu_{\max }$ maximum specific growth rate

$x \quad$ cell density

$x_{i} \quad$ inoculated cell density

$x_{\max } \quad$ maximum cell density

$x_{\text {sum }}$ overall cell density

\section{References}

Brandt, J. E., Briddell, R. A., Srour, E. F., Leemhuis, T. B., Hoffman, R. 1992. Role of c-kit ligand in the expansion of human hematopoietic progenitor cells. Blood 79: 634-641.

Caplan, A. I. 1991. Mesenchymal stem cells. J. Orthopaed. Res. 9: 641-650.

Carnahan, B., Luther, H. A., Wilkes, J. O. 1969. Applied numerical methods. Wiley, New York.

Carow, C. E., Hangoc, G., Broxmeyer, H. E. 1993. Human multipotential progenitor cells (CFU-GEMM) have extensive replating capacity for secondary CFU-GEMM: an effect enhanced by cord blood plasma. Blood 81: 942-949.

Civin, C. I., Strauss, L. C., Brovall, C., Fackler, M. J., Schwartz, J. F., Shaper, J. H. 1984. Antigenic analysis of hematopoiesis. III. A hematopoietic progenitor cell surface antigen defined by a monoclonal antibody raised against KG-Ia cells. J. Immunol. 133: 157-165.

Cronkite, E. P. 1988. Analytical review of structure and regulation of hemopoiesis. Blood Cells 14: 313-328.

Davis, A. A., Temple, S. 1994. A self-renewing multipotential stem cell in embryonic rat cerebral cortex. Nature 372: 263-266.

Dexter, T. M., Allen, T. D., Lajtha, L. G. 1977. Conditions controlling the proliferation of haemopoietic stem cells in vitro. J. Cell Physiol. 91: $335-344$.

Eaves, C. J., Cashman, J. D., Eaves, A. C. 1991. Methodology of longterm culture of human hemopoietic cells. J. Tissue Cult. Meth. 13: $55-62$.

Eaves, C. J., Sutherland, H. J., Udomsakdi, C., Lansdrop, P. M., Szilvassy, S. J., Fraser, C. C., Humphries, R. K., Barnett, M. J., Phillips, G. L., Eaves, A. C. 1992. The human hematopoietic stem cell in vitro and in vivo. Blood Cells 18: 301-307.

Emerson, S. G. 1991. The stem cell model of hematopoiesis, pp. 72-81. In: R. Hoffman et al. (eds.), Hematology: basic principles and practice. Churchill Livingstone, New York.

Greenberger, J. S. 1984. Long-term hematopoietic cultures, pp. 203242. In: D. W. Golde (ed.), Hematopoiesis. Churchill Livingstone, New York.

Hall, P. A., Watt, F. M. 1989. Stem cells: the generation and maintenance of cellular diversity. Development 106: 619-633.

Heimfeld, S., Weissman, I. 1991. Development of mouse hematopoietic lineages. Curr. Topics Dev. Biol. 25: 155-175.

Koller, M. R., Bender, J. G., Miller, W. M., Papoutsakis, E. T. 1993a. Expansion of primitive human hematopoietic progenitors in a perfusion bioreactor system with IL-3, IL-6, and stem cell factor. Bio/ Technology 11: 358-363.

Koller, M. R., Emerson, S. G., Palsson, B. O. 1993b. Large-scale expansion of human stem and progenitor cells from bone marrow mononuclear cells in continuous perfusion cultures. Blood 82: 378-384.

Koller, M. R., Palsson, B. O. 1993. Tissue engineering: reconstruction of human hematopoiesis ex vivo. Biotechnol. Bioeng. 42: 909-930.

Lansdorp, P. M., Dragowska, W. 1992. Long-term erythropoiesis from constant numbers of $\mathrm{CD} 34^{+}$cells in serum-free culture initiated with highly purified progenitor cells from human bone marrow. J. Exp. Med. 175: 1501-1509. 
Mayani, H., Dragowska, W., Lansdorp, P. M. 1993. Cytokine-induced selective expansion and maturation of erythroid versus myeloid progenitors from purified cord blood precursor cells. Blood 81: 3252-3258

Moore, M. A. S. 1991. Clinical implications of positive and negative hematopoietic stem cell regulators. Blood 78: 1-19.

Nakahata, T., Gross, A. J., Ogawa, M. 1982. A stochastic model of self-renewal and commitment to differentiation of the primitive hematopoietic stem cells in culture. J. Cell Physiol. 113: 455-458.

Nielsen, J., Villadsen, J. 1992. Modelling of microbial kinetics. Chem. Eng. Sci. 47: 4225-4270.

Palsson, B. O., Paek, S.-H., Schwartz, R. M., Palsson, M., Lee, G.-M., Silver, S. Emerson, S. G. 1993. Expansion of human bone marrow progenitor cells in a high cell density continuous perfusion system. Bio/Technology 11: 368-372.

Peng, C.-A., Palsson, B. O. 1995. The importance of non-homogeneous concentration distributions near walls in tissue engineered bioreactor. Ind. Eng. Chem. Res. 34: 3239-3245.

Peng, C.-A., Palsson, B. O. 1996. Stem cell replication and differentiation in tissue engineering bioreactors strongly influenced by bioreactor geometry. Biotechnol. Bioeng. 50: 479-492.

Ploemacher, R. E., van der Sluijs, J. P., van Beurden, C. A. J., Baert, M. R. M., Chan, P. L. 1991. Use of limiting dilution type long-term marrow cultures in frequency analysis of marrow-repopulating and spleen colony-forming hematopoietic stem cells in the mouse. Blood 78: $2527-2533$.

Potten, C. S., Loeffler, M. 1987. A comprehensive model of the crypts of the small intestine of the mouse provide insight into the mechanisms of cell migration and proliferation hierarchy. J. Theor. Biol. 127: 381-391.

Potten, C. S., Loeffler, M. 1990. Stem cells: attributes, cycles, spirals, pitfalls and uncertainties. Development 110: 1001-1020.

Potten, C. S., Wichmann, H. E., Loeffler, M., Dobek, K., Major, D. 1982. Evidence for discrete cell kinetic subpopulations in mouse epidermis based on mathematical analysis. Cell Tissue Kinet. 15: 305-320.

Schmitz, S., Franke, H., Brusis, J., Wichmann, H. E. 1993. Quantification of the cell kinetic effects of G-CSF using a model of human granulopoiesis. Exp. Hematol. 21: 755-760.
Schofield, R., Lord, B. I., Kyffin, S., Gilbert, C. W. 1980. Selfmaintenance capability of CFU-S. J. Cell Physiol. 103: 355-362.

Schwartz, R. M., Emerson, S. G., Clarke, M. F., Palsson, B. O. 1991 a. In vitro myelopoiesis stimulated by rapid medium exchange and supplementation with hematopoietic growth factors. Blood $\mathbf{7 8}$ : 3155-3161.

Schwartz, R. M., Palsson, B. O., Emerson, S. G. 1991b. Rapid medium perfusion rate significantly increases the productivity and longevity of human bone marrow culture. Proc. Natl. Acad. Sci. USA 88: $6760-6764$.

Sell, S. 1990. Is there a liver stem cell? Cancer Res. 50: 3811-3815.

Sigal, S. H., Brill, S., Fiorino, A. S., Reid, L. M. 1992. The liver as a stem cell and lineage system. Am. J. Physiol. 263: G139-G148.

Srour, E. F., Brandt, J. E., Briddell, R. A., Grigsby, S., Leemhuis, T., Hoffman, R. 1993. Long-term generation and expansion of human primitive hematopoietic progenitor cells in vitro. Blood 81: 661-669.

Stemple, D. L., Anderson, D. J. 1992. Isolation of a stem cell for neurons and glia from the mammalian neural crest. Cell 71: 973-985.

Sutherland, H. J., Lansdorp, P. M., Henkelman, D. H., Eaves, A. E., Eaves, C. J. 1990. Functional characterization of individual human hematopoietic stem cells cultured at limiting dilution on supportive marrow stromal layers. Proc. Natl. Acad. Sci. USA 87: 3584-3588.

Thorgeirsson, S. S. 1993. Hepatic stem cells. Am. J. Pathol. 142: 13311333.

Till, J. E., McCulloch, E. A., Siminovitch, L. 1964. A stochastic model of stem cell proliferation, based on the growth of spleen-colony forming cells. Proc. Natl. Acad. Sci. USA 51: 29-36.

Vogel, H., Niewisch, H., Matoli, G. 1968. The self-renewal probability of hemopoietic stem cells. J. Cell Physiol. 72: 221-228.

Warner, H. R., Athens, J. W. 1964. An analysis of granulocyte kinetics in blood and bone marrow. Ann. NY Acad. Sci. 113: 523-536.

Wichmann, H. E., Loeffler, M., Schmitz, S. 1988. A concept of hemopoietic regulation and its biomathematical realization. Blood Cells 14: 411-429.

Wright, N. A. 1983. The cell proliferation kinetics of the epidermis, pp. 203-229. In: L. A. Goldsmith (ed.), Biochemistry and physiology of the skin. Oxford University Press, Oxford, UK. 